\title{
Comparison of health-related quality of life in patients with rheumatoid arthritis during conventional or conventional plus biological therapy in Poland
}

This article was published in the following Dove Medical Press journal:

Patient Preference and Adherence

\section{Ewelina Bąk' \\ Czesław Marcisz ${ }^{2}$ \\ Adriana Borodzicz ${ }^{3}$ \\ Danuta Sternal' \\ Sylwia Krzemińska}

'Faculty of Health Sciences, University of Bielsko-Biala, Bielsko-Biala, Poland;

${ }^{2}$ Department of Gerontology and Geriatric Nursing, School of Health Sciences, Medical University of Silesia, Katowice, Poland; ${ }^{3}$ Department of Clinical Nursing Faculty of Health Sciences, Medical University, Wroclaw, Poland
Correspondence: Ewelina Bąk Faculty of Health Sciences, University of Bielsko-Biala, ul. Willowa 2, 43-309 Bielsko-Biala, Poland

Tel +48 338279198

Email ewelina.bak76@wp.pl
Purpose: The main purpose of this study was to compare the level of health-related quality of life (HRQoL) using Nottingham Health Profile (NHP) in Polish patients with rheumatoid arthritis (RA) during therapy applying disease-modifying antirheumatic drugs (DMARDs) with conventional synthetics (csDMARDs) or with csDMARDs in combination with biological drugs (bDMARDs). The second purpose was to analyze the correlation between the domain values of NHP and the demographic and clinical parameters, functional efficiency, and mood. Patients and methods: The studies involved 212 patients with RA, divided into two groups: group I - 126 persons treated using csDMARDs, group II - 86 patients using csDMARDs in combination with bDMARDs. A diagnostic survey was used applying NHP for HRQoL, Beck Depression Inventory (BDI), and Health Assessment Questionnaire (HAQ). The 28-Joint Disease Activity Score (DAS-28) was calculated.

Results: The patients with RA in both studied groups did not differ significantly in terms of all the NHP domains, values of HAQ and BDI. The DAS-28 value, the number of swollen joints, and the duration of morning stiffness were significantly smaller among patients from group II. However, in both groups, the majority of the analyzed components of NHP demonstrated significant correlations with values of HAQ and BDI and some of the domains of NHP - with DAS-28. Conclusion: The level of HRQoL, functional efficiency, and mood are comparable in patients treated conventionally and in combination with biological drugs. The HRQoL level shows correlation with the occurrence of depression symptoms, and the energy level, the sensation of pain, and physical abilities are covariates with daily activities. The intensity of the activity of RA as well as experiencing pain and the duration of morning stiffness is smaller among patients applying csDMARDs plus bDMARDs compared with patients treated only conventionally.

Keywords: patients with rheumatoid arthritis in Poland, DMARDs, biological therapy, healthrelated quality of life, Nottingham Health Profile, Beck Depression Inventory, Health Assessment Questionnaire

\section{Introduction}

Rheumatoid arthritis (RA) is a chronic disease of the connective tissue. The symptoms of the disease are nonspecific arthritis, extraarticular lesions, and organ complications. RA involves a risk of the occurrence of disability or even premature death. ${ }^{1}$ In accordance with the most recent diagnostic-therapeutic standards of the American College of Rheumatology (ACR) and the European League Against Rheumatism (EULAR), it is important to make an appropriate diagnosis and to begin 
therapy applying disease-modifying antirheumatic drugs (DMARDs). ${ }^{2,3}$ The treatment modifying the course of RA includes conventional synthetic drugs (csDMARDs) and biological drugs (bDMARDs). As confirmed by the studies by Marshall et al, ${ }^{4}$ the treatment applying the biological method significantly improved physical and social functioning, reduced the pain sensation and the intensity of morning stiffness, and it improved the patients' well-being. Jørgensen et $\mathrm{al}^{5}$ achieved an improvement of the level of the healthrelated quality of life (HRQoL) in patients with RA after 6 months of bDMARDs treatment in monotherapy.

During the observation of the activity of RA, of its course and of the effectiveness of treatment, besides monitoring changes in the scope of clinical symptoms, biochemical indicators, and imaging indicators, there is also a need to take into consideration the level of the HRQoL in patients with RA. This results from the need for a holistic approach to methods of treatment and from the necessity to monitor patients with RA in the scope of their physical, mental, and social functioning. In the course of RA, patients experience deterioration of the level of the HRQoL, ${ }^{6,7}$ which may be affected by many factors, the most important of which include pain, fatigue, impaired functioning in the family and in the society, the degree of disability, depression, ${ }^{7-9}$ comorbidities, ${ }^{6,10}$ and the type of the applied treatment. ${ }^{11}$ Studies on the HRQoL in patients with RA in Poland were carried out at several research centers. ${ }^{12-15}$ Patients with RA demonstrated a low level of HRQoL in terms of both domains - the physical one and the mental one-determined using the 36 items short-form health survey (SF-36). ${ }^{13,14}$ However, mental functioning was demonstrated to be at a higher level than physical functioning. Also in another study, in patients treated using csDMARDs or bDMARDs, the point values of the physical component summary were significantly lower than the values of the mental component summary and they proved to be comparable in both groups of the studied patients. ${ }^{15}$ Kanecki et al ${ }^{12}$ demonstrated that during the period of a 2-year observation of patients treated with RA, there was a deterioration of social functioning and an improvement of the role of emotional and mental health.

In studies on HRQoL in patients with RA, various diagnostic methods were applied with the use of the Arthritis Impact Measurement Scales, ${ }^{16}$ the Rheumatoid Arthritis Quality of Life instrument, ${ }^{17-19}$ the Health Assessment Questionnaire (HAQ), ${ }^{6,20}$ the Nottingham Health Profile (NHP), ${ }^{8,18,19,21,22}$ EuroQoL-5-Dimensions ${ }^{5,6}$ as well as the SF-36. ${ }^{7,13,23}$ Selecting the method for determining HRQoL and defining the factors that affect the QoL of patients with RA may lead to the selection of the proper treatment process, which would be most beneficial for improving the self-assessment of health of the treated patients.

The main purpose of this study was to compare the level of the HRQoL measured using the NHP in Polish patients with RA during therapy with csDMARDs or with the addition of bDMARDs. The secondary purpose was to analyze the correlation between the values of the NHP domains and the demographic and clinical parameters, functional efficiency, and mood.

\section{Materials and methods}

\section{Participants}

The studies involved 212 patients with RA who were divided into two groups. Group I consisted of 126 persons treated using csDMARDs, including 102 women and 24 men aged 23 to 90 years, mean $\pm \mathrm{SD}=58.4 \pm 11.1$ years. Group II comprised 86 patients treated using csDMARDs plus bDMARDs, including 60 women and 26 men aged 31 to $69(52.4 \pm 10.0)$ years. All the studied subjects were patients of the Silesian Center for Rheumatology, Rehabilitation, and Disability Prevention in Ustron, Poland. The studies were carried out among consecutive hospital inpatients and clinic outpatients with RA in the period from March 2017 to November 2017. The inclusion criteria for the group of studied patients were as follow: the diagnosis of RA based on the ACR/EULAR 2010 diagnostic standards, ${ }^{2}$ age $>18$ years and providing written consent for the study to be performed. The patients from Group I were selected out of 145 randomly chosen patients with RA treated using csDMARDs. The reasons for reducing the number of patients qualified for further studies were a lack of consent of eleven patients and incompletely filled-in surveys (eight patients). The patients from Group II were selected out of 109 randomly chosen patients with RA treated using csDMARDs plus bDMARDs. The consideration of only 86 patients in the studies was related to the lack of written consent of 16 patients and to incompletely filled-in surveys of 7 patients.

Considering the fact that in Poland about $1 \%$ of the population of adults suffer from $\mathrm{RA}^{24}$ with the assumption that the maximum error is $2 \%$ and with a significance level of 0.05 , the minimum sample size is estimated to be 96 persons, which is fewer than 212 patients in this study.

The research was performed with the consent of the Bioethics Committee of the Beskidzka Regional Chamber of Physicians in Bielsko-Biala; the consent was provided during the meeting held on February 16, 2017 (No. of consent $2017 / 02 / 16 / 6)$. All the procedures performed during the 
studies involving human participants were in accordance with the ethical standards of the committee and with the 1964 Declaration of Helsinki. Before participating in the study, every person was informed about its purpose.

The treatment of RA generally involved using DMARDs such as methotrexate (MTX), sulfasalazine, nonsteroidal anti-inflammatory drugs, and glucocorticosteroids (GCS; Group I) and biological therapy which included TNF inhibitors, etanercept, adalimumab, and additionally MTX as well as GCS; Group II).

\section{Methods}

The values of the following biochemical parameters were defined in all the studied patients: the concentration of the C-reactive protein (CRP), creatinine, the activity of alanine transaminase and aspartate transaminase, and the erythrocyte sedimentation rate (ESR). The studied persons were weighed, their body height measured, and the body mass index (BMI) calculated. The Cockroft-Gault formula was used to define the estimated glomerular filtration rate in $\mathrm{mL} /$ hour $/ 1.73 \mathrm{~m}^{2}$. The 28-Joint Disease Activity Score (DAS-28) was also calculated based on the following formula: DAS-28 $=0.56 \sqrt{ }($ TEN28 $)+0.28 \sqrt{ }(\mathrm{SW} 28)+0.36 \mathrm{Ln}$ $(\mathrm{CRP}+1)+0.014(\mathrm{VAS})+0.96,{ }^{25}$ where TEN28 $=$ the number of painful joints out of 28 assessed, SW28 = the number of swollen joints out of 28 assessed, $\mathrm{CRP}=$ the concentration of the CRP in the serum, VAS = the Visual Analog Scale for pain.

All the studied patients were surveyed with the use of the following questionnaires: the patient demographic and clinical data survey, NHP, the Beck Depression Inventory (BDI), and the HAQ. The questionnaires were filled in by the patients themselves during a doctor appointment in the presence of the authors (EB and AB) carrying out the studies. The time needed for completing the survey was 15-20 minutes.

\section{The patient demographic and clinical data survey}

The questionnaire concerning sociodemographic and clinical characteristics included the following parameters: gender, age, BMI, place of residence, education, marital status, professional activity, stimulants used, the number of painful and swollen joints, the duration of morning stiffness, the duration of RA, the duration of biological treatment, comorbidities, and RA drugs used.

\section{Nottingham Health Profile}

The measurement of the assessment of the QoL of patients with RA was performed using the general questionnaire, ie NHP. This questionnaire was developed by British researchers, Hunt et al. ${ }^{21}$ It consists of two parts: the basic one and the supplementary one. The basic part includes 38 simple statements referring to six dimensions of HRQoL, ie, energy level (NHP-EL), pain (NHP-P), emotional reaction (NHP-ER), sleep (NHP-S), social isolation (NHP-SI), and physical abilities (NHP-PA). The supplementary part is composed of seven statements which refer to the influence of the current health status on the following areas of life: paid employment, housework, social life, family life, sex life, hobbies, and holidays. The results of the first part of the questionnaire were calculated using the key for each of the six dimensions separately. The higher the result in the point score, the worse is the QoL in the analyzed area. The Polish adaptation of the NHP was prepared with the consent of the authors of the questionnaire. The internal consistency results obtained with the Polish adaptation of the NHP were similar to the data from the original version. ${ }^{26}$

\section{Beck Depression Inventory}

The BDI is a 21-point screening tool used to assess the severity degree of mood disorder symptoms. The scale composed of 21 questions, which may be scored from 0 to 3 points. The results obtained in the BDI range between 0 and 63 . Higher scores indicate more acute depression symptoms. ${ }^{27}$ The BDI is a standardized questionnaire adjusted to the Polish conditions.

\section{Health Assessment Questionnaire}

The assessment of physical function was performed using the Polish version of the HAQ. ${ }^{20}$ The questions present in this questionnaire refer to everyday functioning, which includes activities (sections) such as dressing, getting up, eating, walking, hygiene, gripping, reaching, and errands and chores. The points collected from all eight HAQ sections, with the consideration of the devices or activities that the patient needed help with, were summed up and divided by 8 . The HAQ result ranged from 0 to 3 . Calculating it was possible only if the patient filled in at least three sections. A value of $\geq 2.25$ indicated a high degree of disability. In literature, the HAQ value exceeding 1 is considered a clinical indicator of disability. ${ }^{28}$

\section{Statistical analysis}

Statistical calculations were performed using the Statistica (version 13; StatSoft, Cracow, Polska) software. The level of statistical significance considered for all the calculations was $P$-value $=0.05$. The type of distribution was verified 
for all the analyzed variables. The Shapiro-Wilk test was applied. The mean, the standard deviation, and the range were calculated for quantitative data. The elements calculated for qualitative variables were the number and the frequency of their occurrence (in \%). Contingency tables and the chi-squared test were used in the analysis of qualitative variables. The Mann-Whitney $U$-test was applied in order to compare quantitative variables in two independent groups characterized by distributions that were not normal. The Kruskal-Wallis test was applied in order to compare the mean values for variables that did not meet the conditions for applying variance analysis. The reliability of the scale was determined by calculating the Cronbach $\alpha$ coefficient. The multiple regression analysis was performed in order to present the influence of sociodemographic and clinical factors on the subjective assessment of the QoL (NHP). The multiple regression model for patients without biological treatment was developed for each of the six analyzed domains of
NHP; these domains were dependent variables. The factors qualified for the analysis as independent variables included age, education, BDI, HAQ, DAS-28, disease duration, the duration of biological treatment, and BMI.

\section{Results}

The sociodemographic, biochemical, and clinical characteristics of the studied persons are presented in Table 1 . Group I significantly differed from Group II in terms of age, education, professional activity, and smoking tobacco. In the patients from Group I, the ESR values, the number of swollen joints, the duration of morning stiffness as well as the VAS and DAS-28 values were higher and the GFR was lower than in the patients from Group II (Table 1). The duration of RA was significantly higher in the patients from Group II $(P<0.05)$.

The mean values in the particular domains of NHP, BDI, and HAQ did not demonstrate statistically significant

Table I Sociodemographic, clinical, and biochemical characteristics in the patients with rheumatoid arthritis

\begin{tabular}{|c|c|c|c|}
\hline Analyzed parameters & Group I $(n=\mid 26)$ & Group II $(n=86)$ & $P$-value \\
\hline Gender: women/men & $102 / 24$ & $60 / 26$ & 0.0613 \\
\hline Age (years) & $59(53,64)$ & $54(46,58)$ & $0.0008^{*}$ \\
\hline $\mathrm{BMI}\left(\mathrm{kg} / \mathrm{m}^{2}\right)$ & $27.0(24.5,29.7)$ & $25.4(22.8,28.3)$ & $0.005^{*}$ \\
\hline Education (n): primary/vocational/secondary/higher & $17 / 35 / 54 / 20$ & $0 / 19 / 36 / 31$ & $<0.0001$ \\
\hline Place of residence $(n)$ : urban/rural area & $91 / 35$ & $62 / 24$ & 0.9836 \\
\hline Marital status $(\mathrm{n}):$ single/married/widow/divorced & $6 / 89 / 23 / 8$ & $7 / 59 / 8 / 12$ & 0.0724 \\
\hline Professional activity(n): yes/no & $33 / 93$ & $34 / 52$ & 0.0191 \\
\hline Smoking tobacco $(n)$ : nonsmoker/smoker & $95 / 31$ & $76 / 10$ & 0.0160 \\
\hline Drinking alcohol $(n)$ : yes/no & $29 / 97$ & $15 / 7 \mid$ & 0.3221 \\
\hline ESR (mm/hour) & $18(13,29)$ & $15(I I, 20)$ & $0.0045^{*}$ \\
\hline $\mathrm{CRP}(\mathrm{mg} / \mathrm{dL})$ & $0.74(0.32,1.3)$ & $0.7 \mathrm{I}(0.6, \mathrm{I} .2)$ & $0.3542 *$ \\
\hline GFR (mL/hour/l.73 m²) & $97(85,116)$ & $103(85,130)$ & $0.1540 *$ \\
\hline AIAT (U/L) & $20(16,28)$ & $21(19,24)$ & $0.3008^{*}$ \\
\hline AspAT (U/L) & $21(17,25)$ & $21(19,24)$ & $0.7456^{*}$ \\
\hline Number of painful joints (n) & $6(3,10)$ & $5(2,7)$ & $0.1174 *$ \\
\hline Number of swollen joints (n) & $4(2,6)$ & $3(2,4)$ & $0.0013^{*}$ \\
\hline Duration of morning stiffness (hour) & $\mathrm{I}(0.3, \mathrm{I})$ & $0.3(0.15,0.85)$ & $0.0424^{*}$ \\
\hline VAS (score) & $60(49,68)$ & $4 I(38,48)$ & $<0.000 I^{*}$ \\
\hline DAS-28 (score) & $3.9(3.4,4.6)$ & $3.6(2.9,4.0)$ & $0.0009 *$ \\
\hline Duration of disease (months) & $72(24,184)$ & $120(84,216)$ & $0.0002^{*}$ \\
\hline Duration of biological treatment (months) & - & $14(12,36)$ & \\
\hline $\begin{array}{l}\text { Comorbidities ( } \mathrm{n}) \text { : diabetes/diseases of the cardiovascular system/ } \\
\text { diseases of the gastrointestinal system/neurologic diseases/diseases } \\
\text { of the skin }\end{array}$ & $7 / 35 / 17 / 15 / 6$ & $2 / 23 / 6 / 7 / 9$ & \\
\hline $\begin{array}{l}\text { Drugs (n): Methotrexate/Sulfasalazine/NSAID/ } \\
\text { Glucocorticosteroids/Etanercept/Adalimumab }\end{array}$ & $119 / 7 / 33 / 34 / 0 / 0$ & $86 / 0 / 0 / 31 / 52 / 34$ & \\
\hline
\end{tabular}

Notes: *Mann-Whitney U-test. Data presented as median (IQR) unless stated otherwise ie, (n).

Abbreviations: AIAT, alanine transaminase; AspAT, aspartate transaminase; BMI, body mass index; CRP, C-reactive protein; DAS-28, 28-Joint Disease Activity Score; ESR, erythrocyte sedimentation rate; GFR, glomerular filtration rate; IQR, interquartile range; NSAIDs, nonsteroidal anti-inflammatory drugs; $P$, statistical significance of differences; VAS, Visual Analog Scale. 
differences between the patients in both study groups (Table 2); only in Group I, there were more problems related to hobbies in NHP due to the health status. The Cronbach $\alpha$ values of NHP, BDI, and HAQ for our results were estimated at the levels $0.88,0.72$, and 0.94 , respectively, for patients in Group I and 0.90, 0.89, and 0.95, respectively, for patients in Group II.

The multiple regression analysis was performed in order to present the relationships between the sociodemographic and clinical factors and the level of the HRQoL. In this model, the NHP domains were the dependent variables. The results are presented in Table 3. Among the patients from Group I, it was demonstrated that each of the analyzed NHP domains was significantly associated with the BDI. NHP-EL, NHP-P, NHP-S, and NHP-PA were associated with the HAQ (Table 3). The value of DAS-28 correlated only with NHP-SI. In the patients in Group II, nearly all the NHP domains, except for NHP-P, were associated with the BDI. HAQ was associated with NHP-EL, NHP-P, and NHP-PA. DAS-28 was associated with NHP-P and NHPPA. Negative correlation was observed between NHP-P and the duration of biological treatment (Table 3). In both of the studied groups, some of the NHP domains were associated with age, BMI and only in Group II with education, the duration of the disease (Table 3).

\section{Discussion}

The present paper analyzed the general HRQoL applying the NHP questionnaire and using the specific HAQ referring to the physical efficiency in everyday functioning of patients with RA during their treatment. The applied questionnaires in the Polish language version were characterized by good internal consistency (reliability) estimated using the Cronbach alpha indicator. Following the consideration of the

Table 2 The quality of life, physical efficiency, and the occurrence of depression in the patients with rheumatoid arthritis

\begin{tabular}{|c|c|c|c|c|}
\hline \multicolumn{2}{|c|}{ Analyzed parameters } & Group I $(n=\mid 26)$ & Group II (n=86) & $P$-value \\
\hline \multicolumn{2}{|l|}{ NHP-EL } & $76(36.8,100)$ & $69.6(36.8,100.0)$ & $0.7147^{*}$ \\
\hline \multicolumn{2}{|l|}{ NHP-P } & $63.5(40.6,81.3)$ & $62.1(40.6,87.1)$ & $0.7890 *$ \\
\hline \multicolumn{2}{|l|}{ NHP-ER } & $80.9(58.7,100)$ & $85.2(63.6,100)$ & $0.2275^{*}$ \\
\hline \multicolumn{2}{|l|}{ NHP-S } & $78.3(34.9,100)$ & $85.7(55.9,100)$ & $0.1968^{*}$ \\
\hline \multicolumn{2}{|l|}{ NHP-SI } & $100(78,100)$ & $100(78.0,100)$ & $0.9197^{*}$ \\
\hline \multicolumn{2}{|l|}{ NHP-PA } & $67.4(54.8,88.5)$ & $67.4(46.6,89.2)$ & $0.9364 *$ \\
\hline \multirow[t]{7}{*}{$\mathrm{NHP}(\mathrm{n}[\%])$} & Paid employment & $44(34.92)$ & $36(42.35)$ & 0.2760 \\
\hline & Housework & $78(61.9)$ & $55(63.95)$ & 0.7618 \\
\hline & Social life & $28(22.22)$ & $27(31.4)$ & 0.1366 \\
\hline & Family life & $19(15.08)$ & $14(16.28)$ & 0.8133 \\
\hline & Sex life & $42(33.33)$ & $38(44.7 I)$ & 0.0957 \\
\hline & Hobbies & $59(46.83)$ & $56(65.88)$ & 0.0061 \\
\hline & Holidays & $50(39.68)$ & $4 \mid(48.24)$ & 0.2189 \\
\hline \multicolumn{2}{|l|}{ HAQ } & $9(5,14)$ & $8(3,14)$ & $0.6808^{*}$ \\
\hline $\begin{array}{l}\text { Dressing and } \\
\text { grooming }\end{array}$ & \multirow[t]{8}{*}{$\begin{array}{l}\text { Without any difficulty/ with some difficulty/ } \\
\text { with much difficulty/unable to do (\%) }\end{array}$} & $32.5 / 29.4 / 38.1 / 0$ & $39.5 / 29.1 / 30.2 / 1.16$ & 0.3305 \\
\hline Getting up & & $30.2 / 34.1 / 34.9 / 0.8$ & $41.9 / 24.4 / 33.7 / 0$ & 0.1975 \\
\hline Eating & & $38.1 / 38.1 / 22.2 / 1.6$ & $41.9 / 37.2 / 17.4 / 3.5$ & 0.6724 \\
\hline Walking & & $41.3 / 33.3 / 24.6 / 0.8$ & $45.4 / 23.3 / 31.4 / 0$ & 0.2711 \\
\hline Hygiene & & $29.4 / 22.2 / 40.5 / 7.9$ & $33.7 / 9.3 / 45.4 / 11.6$ & 0.0848 \\
\hline Reach & & $20.6 / 29.4 / 43.7 / 6.4$ & $25.6 / 32.6 / 34.9 / 7.0$ & 0.5718 \\
\hline Grip & & $29.4 / 10.3 / 57.9 / 2.4$ & $33.7 / 7.0 / 55.8 / 3.5$ & 0.7475 \\
\hline Other activities & & $25.4 / 34.9 / 32.5 / 7.1$ & $38.4 / 19.8 / 36.1 / 5.8$ & 0.0615 \\
\hline \multicolumn{2}{|c|}{ HAQ standardized } & $\mathrm{I} .25(0.625, \mathrm{I} .75)$ & $\mathrm{I}(0.375, \mathrm{I} .75)$ & $0.6808^{*}$ \\
\hline \multicolumn{2}{|l|}{ BDI (score) } & $14(9,22)$ & $12.5(6,20)$ & $0.2529 *$ \\
\hline Depression (\%) & Mild/moderate/without depression & $52.4 / 9.5 / 38.1$ & $47.7 / 5.8 / 46.5$ & 0.3677 \\
\hline
\end{tabular}

Notes: *Mann-Whitney U-test. Data presented as median (IQR) unless stated otherwise ie, (n).

Abbreviations: BDI, Beck Depression Inventory; EL, energy level; ER, emotional reaction; HAQ, Health Assessment Questionnaire; IQR, interquartile ranges; NHP, Nottingham Health Profile; P, pain; P, statistical significance of differences; PA, physical abilities; S, sleep; SI, social isolation. 


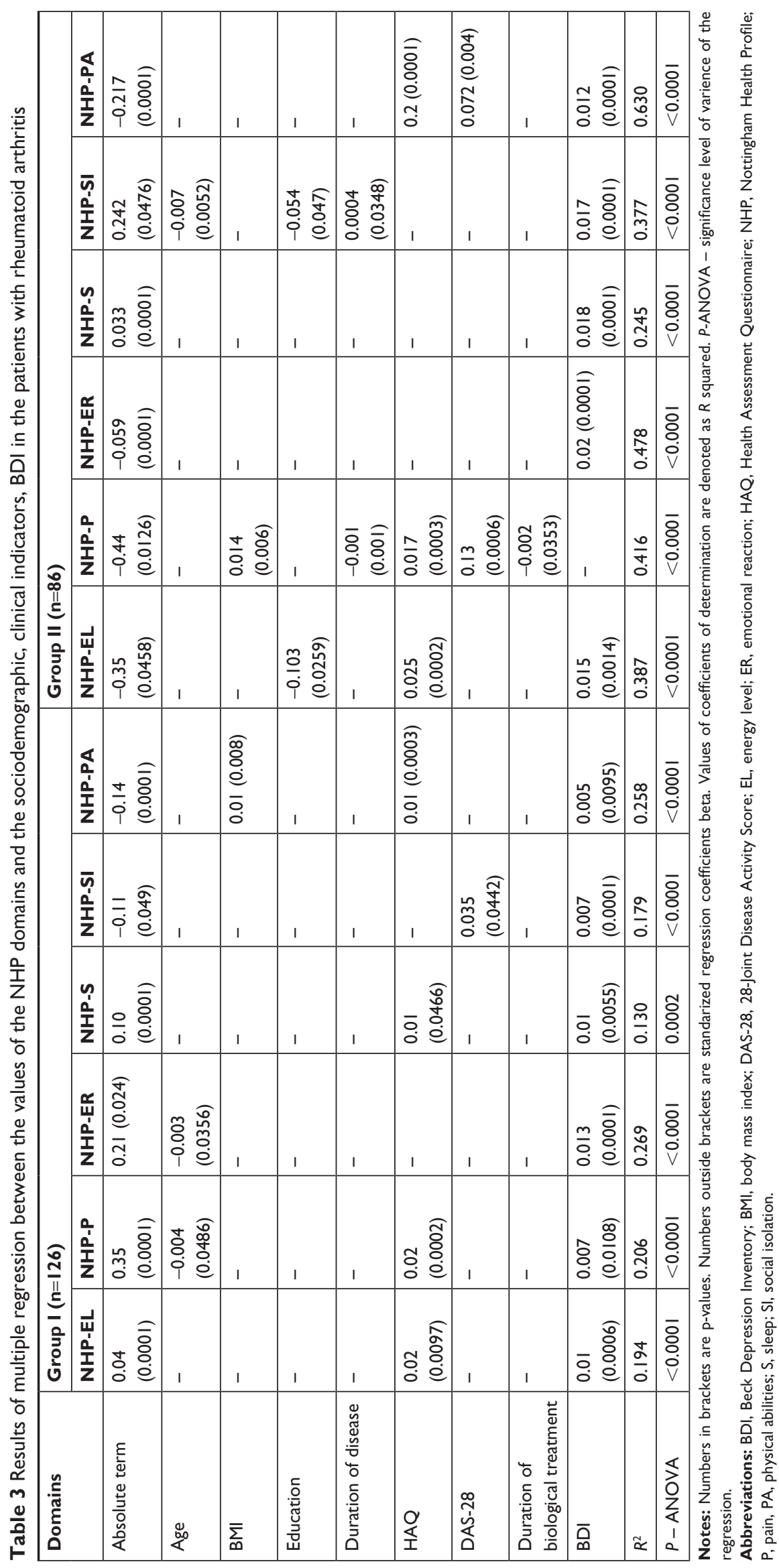


treatment method and dividing the patients into those treated applying csDMARDs and those treated with the addition of bDMARDs, it was demonstrated that the method of treatment of the disease does not seem to lead to a differentiation of the level of declared HRQoL and of the physical efficiency among the studied patients in Poland. It should be stressed, however, that the research groups varied in terms of, among others, age, education, the vocational level, and the onset of the disease, which might have influenced the modification of the HRQoL level. In multiple regression research it has been repeatedly demonstrated that those factors correlate with one or two NHP domains only. It may thus be suggested that the influence of the said factors on HRQoL in the researched patients was small. Irrespective of the method of treatment, the patients with RA demonstrated the lowest level of the QoL in the scope of the energy and pain domains and the highest one in the social isolation and emotional reaction domains. This may mean that the most nagging symptom of RA was experiencing pain and the weakening of life energy, while the best tolerated element was coping with emotions and problems related to social isolation. Similar study results were obtained by Uutela et $\mathrm{al}^{22}$ who, among Finnish patients with RA, demonstrated lowering of the energy level and of physical efficiency as well as higher intensity of pain, whereas, in their studies, the NHP scores for sleep, emotional reaction, and social isolation did not differ from those of the compared healthy persons (controls). Also Munchey and Pongmesa ${ }^{29}$ noticed that among the patients with RA, the most frequently reported problems were difficulties with moving and the discomfort related to experiencing pain. Still, it was revealed in those studies that the value of the DAS-28 and the components of this indicator, ie, the intensity of pain examined using the VAS and the number of swollen joints, were lower among patients receiving combined treatment than among the patients using conventional treatment. Similar conclusions were reached by Inotai et al, ${ }^{11}$ who also demonstrated lower activity of RA and lower pain intensity in patients treated biologically in reference to those treated conventionally. In our own studies, the duration of morning stiffness was shorter among patients treated with the addition of a biological drug. An improvement in morning stiffness resulting from biological treatment of patients with RA was also described by other authors. ${ }^{4}$ It was found that the DAS-28 indicator demonstrated positive correlation with the components of the QoL, namely with NHP-EL, NHP-P, and NHP-PA only in the group of patients receiving combination treatment. It may be assumed that adding biological treatment to the conventional one leads to the weakening of the activity of RA and, in convergence with that, to the reduction of the sensation of pain and to an improvement in terms of energy and physical efficiency. An improvement in terms of physical function and well-being as well as a reduction of pain after biological treatment was observed by Marshall et al. ${ }^{4}$

In the supplementary part of the NHP which includes areas of life such as paid employment, housework, social life, family life, sex life, hobbies, and holidays, no significant differentiation was observed among the patients with RA depending on the type of the pharmacotherapy applied. This may mean that adding biological drugs to conventional treatment did not significantly modify the assessed areas of life of patients with RA.

The studies performed using the HAQ, referring to everyday functioning that includes activities such as dressing, getting up, eating, walking, hygiene, gripping, reaching, and errands and chores, also provided results that were comparable in both of the treated groups of Polish patients with RA. These results were convergent with those obtained in the studies performed by Wysocka-Skurska et al, ${ }^{15}$ who - in Polish population of patients with RA treated conventionally or biologically - did not observe differences in the scope of the daily activities mentioned. It must, however, be pointed out, that in our own studies in both the studied groups, the correlation (determined in multiple regression) with the level of everyday physical efficiency of patients was demonstrated by the values of NHP-EL, NHP-P, and NHP-PA, so the lowering of physical efficiency was accompanied by the deterioration of the QoL in the scope of the mentioned domains irrespective of the method of treatment. Similar observations were made by Uutela et al, ${ }^{22}$ Garip et al, ${ }^{18}$ and Sivas et al, ${ }^{8}$ who, among patients with RA, also demonstrated a positive direction of covariance of the level of the QoL (analyzed using the NHP) with the degree of everyday physical efficiency according to the HAQ. In other studies, the QoL of patients with RA determined using the SF-36 and EQ-5D questionnaires also correlated with the HAQ values. ${ }^{15,29}$

Examining the mood using the BDI and detecting mild and moderate depression allowed to conclude that the results obtained by us in this scope proved to be comparable in both groups of the treated patients with RA. It should be noted that in each of the examined patient groups, regardless of the treatment method, mild depression was observed in about half of the patients. The multiple regression analysis demonstrated significant correlations between the BDI and all the domains of the NHP among the patients treated 
conventionally and almost all the domains - except for NHP-P - among the patients undergoing combined treatment. Literature reports indicate that among patients with RA treated conventionally, all the NHP domains demonstrated significant correlation with the value of the $\mathrm{BDI}^{8}$ or with the Hospital Anxiety and Depression Scale (HADS), ${ }^{9}$ whereas Wysocka-Skurska et al ${ }^{15}$ observed high correlation between the QoL (examined using SF-36) and HADS in patients treated using both the conventional and the combination methods. Our own studies and the ones cited above indicate that the occurrence of depression was a covariate with the deterioration of HRQoL in patients with RA irrespective of the method of treatment.

HRQoL studies of patients with RA carried out by the authors are innovative, as they consider the basic and complementary dimensions of the QoL of Polish patients, treated conventionally and with the addition of biological drugs, in combination with symptoms of depression, disease activity, daily functioning, and sociodemographic indicators. Research of such a wide range has not been carried out in Poland so far.

The limitations present in this paper include the following: firstly, the application of the NHP - a tool that is not RA specific - may provide a quality-of-life-defining result which does not fully reflect the influence of this disease, but also that of the comorbidities. Nevertheless, the obtained results allow for the comparison of the QoL of patients differentiated in terms of the method of treatment. Secondly, the studies were performed during treatment without dynamic observation. It is, therefore, recommended to carry out studies related to the QoL and to everyday activities before undertaking pharmacological treatment of RA and to repeat these studies during the applied therapy, taking into consideration the biological treatment. Thirdly, the QoL and physical efficiency were analyzed only among the patients of a closed rheumatological center, so these patients were not representative of all patients with RA which would require the extension of the studies so as to also include outpatients. Fourthly, the studied groups of patients with the division into those who received and those who did not receive biological treatment, differed in terms of the mean age, which resulted from the adopted requirement for the random selection of persons for the studies. It should, however, be noted that in the multiple regression analysis, the age of the studied patients demonstrated covariance with the values of the NHP-P and NHP-ER domains in the group of patients who did not undergo biological treatment and with NHP-SI in the group that received biological treatment.

\section{Conclusion}

The level of the HRQoL, functional efficiency, and mood are comparable in patients with RA treated conventionally and in combination with the addition of a biological drug. In patients with RA during conventional treatment and those treated with the addition of a biological drug, HRQoL levels correlate with the occurrence of depression symptoms, and the energy level, pain, and physical abilities are covariates with everyday activities. The intensity of the activity of RA as well as experiencing pain and the duration of morning stiffness is lesser among patients applying a combination of conventional and biological treatment compared with patients treated only conventionally.

\section{Ethics approval and consent to participate}

All the procedures performed in the studies involving human participants were in accordance with the ethical standards of the Bioethics Committee of the Beskidzka Regional Chamber of Physicians in Bielsko-Biala; the consent was provided during the meeting held on February 11, 2016 (No of the consent 2016/02/11/1). Written consent was obtained from all the individual participants included in the study.

\section{Disclosure}

The authors report no conflicts of interest in this work.

\section{References}

1. Filipowicz-Sosnowska A, Stanisławska-Biernat E, ZubrzyckaSienkiewicz A. Rheumatoid arthritis. Reumatologia. 2004;42(Suppl 1): 3-16. Polish.

2. Aletaha D, Neogi T, Silman AJ, et al. 2010 Rheumatoid arthritis classification criteria: an American College of Rheumatology/European League against rheumatism collaborative initiative. Arthritis Rheum. 2010;62(9):2569-2581.

3. Smolen JS, Landewé R, Bijlsma J, et al. EULAR recommendations for the management of rheumatoid arthritis with synthetic and biological disease-modifying antirheumatic drugs: 2016 update. Ann Rheum Dis. 2017;76(6):960-977.

4. Marshall NJ, Wilson G, Lapworth K, Kay LJ. Patients' perceptions of treatment with anti-TNF therapy for rheumatoid arthritis: a qualitative study. Rheumatology. 2004;43(8):1034-1038.

5. Jørgensen TS, Turesson C, Kapetanovic M, et al. EQ-5D utility, response and drug survival in rheumatoid arthritis patients on biologic monotherapy: a prospective observational study of patients registered in the South Swedish SSATG registry. PLoS One. 2017;12(2):e169946.

6. Nakajima A, Inoue E, Shimizu Y, et al. Presence of comorbidity affects both treatment strategies and outcomes in disease activity, physical function, and quality of life in patients with rheumatoid arthritis. Clin Rheumatol. 2015;34(3):441-449.

7. Senra H, Rogers H, Leibach G, et al. Health-related quality of life and depression in a sample of Latin American adults with rheumatoid arthritis. Int J Rheum Dis. 2017;20(11):1684-1693.

8. Sivas F, Erçin O, Tanyolaç O, Barça N, Aydoğ S, Özoran K. The Nottingham health profile in rheumatoid arthritis: correlation with other health status measurements and clinical variables. Rheumatol Int. 2004; 24(4):203-206. 
9. Nas K, Sarac AJ, Gur A, et al. Psychological status is associated with health related quality of life in patients with rheumatoid arthritis. J Back Musculoskelet Rehabil. 2011;24(2):95-100.

10. Garip Y, Eser F, Bodur H. Comorbidities in Turkish patients with rheumatoid arthritis: association with the health-related quality of life in terms of disease activity, functional and radiological status, severity of pain, and social and emotional functioning. Acta Reumatol Port. 2016; 41(4):344-349.

11. Inotai A, Rojkovich B, Fülöp A, Jászay E, Ágh T, Mészáros A. Healthrelated quality of life and utility in patients receiving biological and non-biological treatments in rheumatoid arthritis. Rheumatol Int. 2012; 32(4):963-969.

12. Kanecki K, Tyszko P, Wisłowska M, Łyczkowska-Piotrowska J. Preliminary report on a study of health-related quality of life in patients with rheumatoid arthritis. Rheumatol Int. 2013;33(2):429-434.

13. Pytel A, Demczyszak I, Sutkowska E, Rosińczuk J, Kuberka I, Kołtuniuk A. Knowledge and selected variables as determinants of the quality of life and general health of patients with rheumatoid arthritis. Adv Clin Exp Med. 2017;26(9):1411-1418.

14. Wysocka-Skurska I, Sierakowska M, Kułak W. Evaluation of quality of life in chronic, progressing rheumatic diseases based on the example of osteoarthritis and rheumatoid arthritis. Clin Interv Aging. 2016;11: 1741-1750. Polish.

15. Wysocka-Skurska I, Sierakowska M, Sierakowski S. The assessment of the quality of life of patients with rheumatoid arthritis depending on the applied pharmacological therapy. Reumatologia. 2012;50(1):16-23.

16. Meenan RF, Gertman PM, Mason JH. Measuring health status in arthritis. The arthritis impact measurement scales. Arthritis Rheum. 1980;23(2):146-152.

17. de Jong Z, van der Heijde D, Mckenna SP, Whalley D. The reliability and construct validity of the RAQoL: a rheumatoid arthritis-specific quality of life instrument. Rheumatology. 1997;36(8):878-883.

18. Garip Y, Eser F, Bodur H. Health-related quality of life in rheumatoid arthritis: comparison of RAQoL with other scales in terms of disease activity, severity of pain, and functional status. Rheumatol Int. 2011; 31(6):769-772.

19. Zlatkovic-Svenda M, Rouse M, Radak-Perovic M, et al. Adaptation and validation of the rheumatoid arthritis quality of life (RAQoL) questionnaire for use in Serbia. Rheumatol Int. 2017;37(4):641-646.
20. Fries JF, Spitz P, Kraines RG, Holman HR. Measurement of patient outcome in arthritis. Arthritis Rheum.1980;23(2):137-145.

21. Hunt SM, Mckenna SP, Mcewen J, Williams J, Papp E. The Nottingham health profile: subjective health status and medical consultations. Soc Sci Med A. 1981;15(3):221-229.

22. Uutela T, Hakala M, Kautiainen H. Validity of the Nottingham Health Profile in a Finnish out-patient population with rheumatoid arthritis. Rheumatology (Oxford). 2003;42(7):841-845.

23. Ware JE, Sherbourne CD. The MOS 36-item short-form health survey (SF-36). I. Conceptual framework and item selection. Med Care. 1992; 30:473-483.

24. Tłustochowicz W, Brzosko M, Filipowicz-Sosnowska A. The opinion of the team of experts of the National consultant on rheumatology regarding the diagnostics and therapy of rheumatoid arthritis. Reumatologia. 2008;46(3):111-114. Polish.

25. Wells G, Becker JC, Teng J, et al. Validation of the 28-joint disease activity score (DAS28) and European League against rheumatism response criteria based on $\mathrm{C}$-reactive protein against disease progression in patients with rheumatoid arthritis, and comparison with the DAS28 based on erythrocyte sedimentation rate. Ann Rheum Dis. 2009;68(6):954-960.

26. Wrzesniewski K. Examining the subjective health status using the Polish adaptation of the Nottingham Health Profile. In: Karski JB, Kirschner H, Leowski J, editors. Contemporary Needs and Possibilities of Health Measurement. Warszawa, Poland: Ignis; 1997: 37-41. Polish.

27. Beck AT, Steer RA, Brown GK. Manual for the Beck Depression Inventory-II. San Antonio, TX, USA: Psychological Corporation; 1996.

28. Sokka T, Krishnan E, Häkkinen A, Hannonen P. Functional disability in rheumatoid arthritis patients compared with a community population in Finland. Arthritis Rheum. 2003;48(1):59-63.

29. Munchey R, Pongmesa T. Health-related quality of life and functional ability of patients with rheumatoid arthritis: a study from a tertiary care hospital in Thailand. Value Health Reg Issues. 2018;15:76-81.
Patient Preference and Adherence

\section{Publish your work in this journal}

Patient Preference and Adherence is an international, peer-reviewed, open access journal that focuses on the growing importance of patient preference and adherence throughout the therapeutic continuum. Patient satisfaction, acceptability, quality of life, compliance, persistence and their role in developing new therapeutic modalities and compounds to optimize

\section{Dovepress}

clinical outcomes for existing disease states are major areas of interest for the journal. This journal has been accepted for indexing on PubMed Central. The manuscript management system is completely online and includes a very quick and fair peer-review system, which is all easy to use. Visit http://www. dovepress.com/testimonials.php to read real quotes from published authors. 